\title{
Discontinuous change in the smectic layer thickness in ferrielectric liquid crystals
}

\author{
V. P. Panov and J. K. Vij* \\ Department of Electronic and Electrical Engineering, Trinity College, University of Dublin, Dublin 2, Ireland \\ Yu. P. Panarin \\ Department of Electronic and Electrical Engineering, Trinity College, University of Dublin, Dublin 2, Ireland \\ and Department of Electronics, Dublin Institute of Technology, Dublin, Ireland \\ C. Blanc and V. Lorman \\ UMR 5587, CNRS, Université Montpellier II, Place E. Bataillon, 34095 Montpellier, France \\ J. W. Goodby \\ Department of Chemistry, University of York, York, YO10 5DD, United Kingdom \\ (Received 10 April 2006; revised manuscript received 5 January 2007; published 9 April 2007)
}

\begin{abstract}
The temperature dependence of the thickness of thick free-standing films is studied using a high-resolution film thickness measurement technique. A small discontinuity in the temperature dependence of the smectic layer thickness at every phase transition between ferro-, ferri-, and antiferroelectric phases is observed. We show that the major contribution to it arises from a change in the smectic tilt angle.
\end{abstract}

DOI: 10.1103/PhysRevE.75.042701

PACS number(s): 64.70.Md, 61.30.Cz, 61.30.Eb, 77.80.-e

Smectic liquid crystals form layered liquid crystalline phases. In tilted smectic phases the average direction of the elongated molecules is tilted by an angle with respect to the layer normal. In chiral smectics the layers are spontaneously polarized in the plane of the layer and perpendicular to the direction of the tilt $[1,2]$. A number of intermediate ferrielectric phases had been discovered between ferroelectric smectic $C^{*}\left(\mathrm{Sm}^{*}\right)$ and antiferroelectric smectic $C_{A}^{*}\left(\mathrm{Sm}_{A}^{*}\right)[3]$. The general and well-established phase sequence on cooling from paraelectric smectic $A(\operatorname{Sm} A)$ is as follows: ferroelectric $\mathrm{SmC}^{*}$; four-layer ferrielectric $\mathrm{SmC}_{4}^{*}$ (also known as $\mathrm{SmC}_{F I 2}^{*}$ [4] or AF [3]); three-layer ferrielectric $\mathrm{SmC}_{3}^{*}$ (or $\mathrm{SmC}_{F I 1}^{*}[4]$ or $\mathrm{SmC}_{\gamma}^{*}[3]$ ), and finally two-layer antiferroelectric $\mathrm{SmC}_{2}$ or $\mathrm{SmC}_{A}^{*}$. Theory [5] predicts a number of intermediate phases in a narrow temperature range between $\mathrm{SmC}_{\gamma}^{*}$ and $\mathrm{SmC}_{A}^{*}$. Some of these have been reported experimentally (see, for example,[6-10]).

The phase transition from orthogonal $\mathrm{Sm} A$ to tilted $\mathrm{Sm} C^{*}$ is associated with the appearance of the pseudovector order parameter $\xi=\left(\mathbf{n} \cdot \mathbf{k}_{0}\right)\left[\mathbf{n} \times \mathbf{k}_{0}\right]$, where $\mathbf{k}_{0}$ is the smectic layer normal, and the director is specified as $n$ $=(\sin \theta \cos \phi, \sin \theta \sin \phi, \cos \theta)$ in terms of the tilt angle $\theta$ and the azimuthal angle $\phi$. This transition can be described within the mean-field theory by taking the free energy in Landau-Ginzburg form [11]:

$$
F=a \tau \xi^{2}+b \xi^{4}+O\left(\xi^{6}\right)
$$

where $\tau=\left(T-T_{C}\right) / T_{C}$ is the dimensionless deviation of the temperature $T$ from the $\mathrm{Sm} A-\mathrm{Sm} C^{*}$ transition temperature $T_{C} ; a$ and $b$ are the Taylor expansion coefficients. The more advanced approach has suggested $b$ to be a function of temperature as well [12].

\footnotetext{
*Electronic address: jvij@tcd.ie
}

While being mathematically simple, the mean-field theory is a powerful technique to describe the properties of new experimental systems and phases. However, it is based on an approximation of the thermodynamic properties of systems where the order parameter is spatially constant. When modeling the ferro-, ferri-, and antiferroelectric phases the variation in the azimuthal angle $\phi$ between the neighboring layers is usually considered as the main distinctive feature in the characterization of the molecular arrangement. This feature is ignored in the mean-field approach and the value of the tilt angle as a function of the temperature but not of a particular phase is taken into account. Meanwhile in many experimental works one can see a clear jump in the tilt angle on cooling from the $\mathrm{SmC}^{*}$ phase down to a low-temperature phase $[6,13-15]$. These results cannot be described by the classical mean-field theory.

In this paper we show that the jumps in the tilt angle happen not only at this, but at every phase transition between the ferrielectric phases, and analyze their influence on the optical thickness. Recently we developed a high-resolution free-standing film (FSF) thickness measurement technique which is based on using a fiber-optic spectrometer (AvaSpec2048). The liquid crystalline films were stretched across a 3-mm-diameter hole drilled in a 0.3 -mm-thick steel plate mounted within a hot stage with a temperature resolution of $0.01{ }^{\circ} \mathrm{C}$. A special algorithm gives fast and reliable fitting of the interference fringes formed on reflection from the film in order to obtain the film thickness. The optical thickness $n d$ (where $n$ is an effective refractive index and $d$ is the mechanical film thickness) is measured in the range of a few tens of micrometers with a resolution better than $0.01 \%$. In the case when the selective reflection band lies within the spectrometer range $(400-1100 \mathrm{~nm}$ in our case), the helical pitch and the thickness of the film are obtained in a single experiment. This gives valuable information for characterizing the phases in the liquid crystalline materials under investigation. 


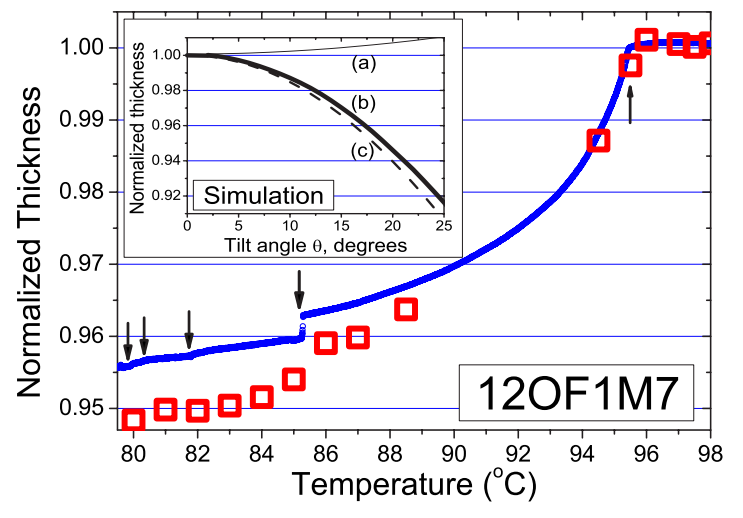

FIG. 1. (Color online) Comparison of the thickness measurements by the x-ray (squares) and optical FSF thickness (circles) techniques. Inset: relative influence of the layer shrinkage and the change in the refractive index on the results of the optical film thickness measurements. Curve $a$, no layer shrinkage considered; curve $b$, simulation of a realistic material with layer shrinkage and molecular dielectric anisotropy, and curve $c$, rigid rod approximation without considering a change in the refractive index.

In our experiment the optical thickness of a thick (over 10000 layers) FSF is measured. For such a thick film, the surface effects can easily be neglected but we can still achieve a high-quality homeotropic alignment. In order to rule out the thickness change due to a possible destruction of a few layers during the experiment, the measurements were performed during both heating and cooling with a rate as slow as $0.01{ }^{\circ} \mathrm{C} / \mathrm{min}$. The measurements were performed using nonpolarized light incident along the layer normal. Therefore only the components of the biaxial refractive index tensor depending on the tilt angle $\theta$ of the long molecular axis can cause a detectable change in the effective refractive index $n$ and affect the results of our measurements.

A change in the optical thickness can appear as a result of two reasons: (i) a change in the mechanical thickness $d$ of the film and (ii) a change in the effective refractive index $n$ of the liquid crystal. In order to estimate the contribution of these two factors we have performed numerical simulations using Berreman's $4 \times 4$ matrix method [16]. The $4 \times 4$ matrix method was used to simulate spectra of the smectic films with different tilt angles and unit cell structures. The obtained theoretical curves were treated with the same algorithm as the experimental data to ensure that the output has exactly the same meaning as for the experiment. The simulation shows that the possible influence of the azimuthal molecular structure of an intermediate phase on the thickness obtained from the interference pattern of nonpolarized light propagating along the layer normal is negligible.

The results of the simulation are shown on the inset of Fig 1. The thick line $b$ shows the dependence of the measured optical thickness $n d$ on the tilt angle for a rigid rod approximation of the molecular length. The parameters used for the simulations are as follows: mechanical layer thickness $d$ $=d_{0} \cos (\theta)$, dielectric tensor components $\epsilon_{11}=\epsilon_{22}=2.0, \epsilon_{33}$ $=2.54$. The tensor components here are related to a single layer and do not change with phase transitions. The effect of a temperature change on the components of the dielectric anisotropy is gradual and can easily be neglected over a narrow range of temperatures involved under discussion. Therefore the effective refractive index $n$ is a function of the tilt angle $\theta$ only. The dashed line $c$ shows the corresponding changes of the mechanical film thickness normalized using the effective refractive index in the $\operatorname{Sm} A$ phase $\left[n_{0} d\right.$ $\left.=n_{0} d_{0} \cos (\theta)\right]$. As an illustration we also provide a hypothetical change of the optical film thickness in the absence of the mechanical layer shrinkage [i.e., $d=d_{0}=$ const. See Fig 1(a)]. The index 0 here means that the parameter corresponds to the $\operatorname{Sm} A$ phase with zero tilt angle $\theta$. One can see that, for the given dielectric anisotropy, the change in the optical film thickness due to a change in the effective refractive index has been found to have the opposite trend compared to that observed experimentally.

We also compared our data with the smectic layer thickness obtained by the $\mathrm{x}$-ray scattering technique carried out at the $\mathrm{Cu} K \alpha$ wavelength $(\lambda=0.154 \mathrm{~nm})$ (University of Montpellier II, France). For the studied liquid crystalline materials the normalized value of the optically measured film thickness departs from the mechanical film thickness by 10-15\% of the total change in thickness when the tilt angle reaches $25^{\circ}$. This conclusion is in good agreement with our results given in the main part of Fig. 1. The small circles represent the results of the optical film thickness measurements for 12OF1M7, while the large squares show the normalized smectic layer thickness obtained using the x-ray scattering technique. One can see that on cooling the sample in $\mathrm{SmC} \mathrm{C}^{*}$ the x-ray data depart from the optical ones by $10-15 \%$ of the total layer shrinkage as also predicted by our simulations. Therefore we conclude that the main contribution to the change in the optical thickness observed experimentally is a change in the mechanical thickness of the film resulting from a change in the tilt angle. This has also been confirmed by microscopic observations of the meniscus of a free-standing film, which has to absorb the excess of the material resulting from the change in thickness. The x-ray results also confirm a jump in the layer thickness due to the change in the tilt angle at the $\mathrm{SmC}^{*}-\mathrm{AF}$ phase transition. However, the simple $\mathrm{x}$-ray technique is not able to resolve the smaller jumps leaving a niche for implementation of our optical technique.

To describe the detailed structure of the intermediate phases we use the same notation as in [18], i.e., the fourlayer phase has the azimuthal angles of the molecules in its unit cell defined as $0, \delta_{2}, 180^{\circ}, 180^{\circ}+\delta_{2}$, while the threelayer phase has $-\delta_{1} / 2, \delta_{1} / 2,180^{\circ}$. Here $\delta_{1}=\delta_{2}=0$ produces the ideal Ising model, while $\delta_{1}=90^{\circ}$ and $\delta_{2}=120^{\circ}$ correspond to the uniaxial clock model. The values of the distortion angles $\delta$ were recently measured by many groups [17-23]. In most experiments $\delta_{1} / 2$ does not exceed $45^{\circ}$ and $\delta_{2}$ is normally less than $30^{\circ}$. Therefore as a rough first-order approximation we can consider a structure where the molecules in the neighboring layers are either synclinic or anticlinic.

From Fig. 2 it is clear that the tilt angle (and therefore the thickness) in a particular layer depends not only on the temperature (as in the conventional mean-field theory) but also on the relative azimuthal orientation [synclinic (S) or anticlinic (A)] of the adjacent layers. Let us introduce the orientational parameter $\delta d(T)$ as the difference between the thick- 


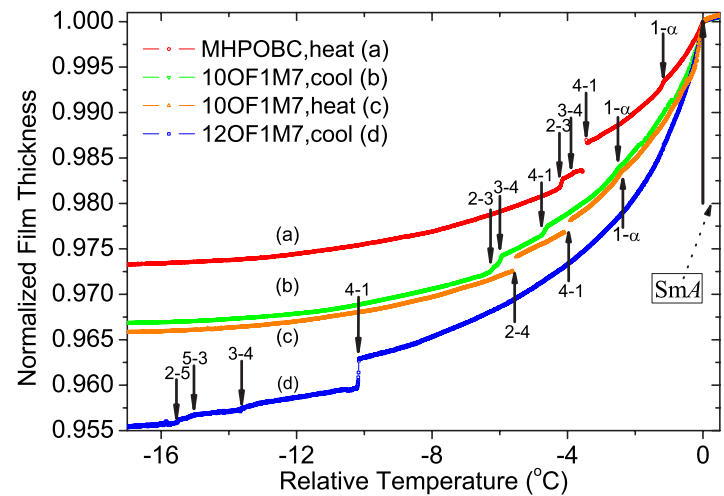

FIG. 2. (Color online) Normalized film thickness vs temperature shows a stepwise change in the thickness at the phase transition. The arrows are marked according to the number of layers in the unit cells of the phases (e.g., 1- $\alpha$ corresponds to the $\mathrm{SmC}^{*}-\mathrm{SmC}_{\alpha}^{*}$ transition, 2-4 to the $\mathrm{SmC}_{A}^{*}$ - $\mathrm{AF}$ transition, etc.).

nesses of synclinic and anticlinic layers $d_{S}(T)-d_{A}(T)$. Since the measured thickness of an $N$-layer phase is the sum of the thicknesses of a large number of layers, it can be presented as $d_{N}=d_{A}(T)+\delta d(T) q_{T}(N)$, where $q_{T}(N)$ is a fraction of synclinic ordering in the $N$-layer phase: $S /(S+A)$ and $S$ and $A$ are the number of synclinic and anticlinic arrangements in the unit cell of an intermediate phase. While this equation is just a rough approximation, it is in good agreement with experimental data shown in Fig. 2. It also demonstrates that our method can be used to find the intermediate phases and determine their unit cell periodicity. Accordingly, the phase observed in 120F1M7 [6] in between two and three layer phases is estimated to be a five layer phase.

The only exception is the transition between the $\mathrm{SmC}$ and $\operatorname{SmC}_{\alpha}^{*}$ phases where there is no clear preference toward synclinic or anticlinic arrangement due to extremely short values of the helical pitch. Moreover, in the case of $12 \mathrm{OF} 1 \mathrm{M} 7$ this transition is not detectable by our technique since it has proven to be continuous [24].

The temperature dependence of the optical film thicknesses for a number of different materials that exhibit intermediate ferrielectric subphases between ferroelectric $\left(\mathrm{SmC}{ }^{*}\right)$ and antiferroelectric $\left(\mathrm{SmC}_{A}^{*}\right)$ phases have been obtained. The temperature dependencies of the optical thickness for three compounds with the following acronyms and phase sequences are presented in Fig. 2. For MHPOBC on heating, as shown in curve $a$,

$$
\begin{gathered}
\mathrm{Sm}_{A}^{*} \stackrel{124.1}{\longrightarrow} \mathrm{Sm}_{\gamma}^{*} \stackrel{124.5}{\longrightarrow} \mathrm{AF} \stackrel{124.9}{\longrightarrow} \mathrm{Sm} C^{*} \\
\stackrel{127.1}{\longrightarrow} \mathrm{Sm}_{\alpha}^{*} \stackrel{128.3}{\longrightarrow} \mathrm{Sm} A^{*} .
\end{gathered}
$$

For $10 \mathrm{OF} 1 \mathrm{M} 7$ on cooling, curve $b$, and on heating curve $c$, heating Fig. 2(c)

$$
\mathrm{Sm}_{A}^{*} \underset{82.1}{\leftrightarrows} \mathrm{Sm}_{\gamma} C_{82.1}^{*} \underset{83.6}{\leftrightarrows} \mathrm{AF} \underset{85.3}{\leftrightarrows} \mathrm{Sm} C^{*} \underset{87.7}{\leftrightarrows} \mathrm{Sm} C_{\alpha}^{*} \underset{87.7}{\leftrightarrows} \mathrm{Sm} A^{*}
$$

For (S)-12OF1M7 on cooling, curve $d$,

$$
\begin{gathered}
\mathrm{Sm}_{A}^{*} \stackrel{77.49}{\longleftarrow} \mathrm{Sm}_{5 l r}^{*} \stackrel{78.3}{\longleftarrow} \mathrm{Sm}_{\gamma}^{*} \stackrel{79.8}{\longleftarrow} \mathrm{AF} \\
\longleftarrow \mathrm{Sm}^{*} \longleftarrow{ }^{\frac{93.5}{\longleftarrow}} \mathrm{Sm}^{*} .
\end{gathered}
$$

One clearly sees a stepwise increase in the optical film thickness at every transition in the phase sequences of the studied materials.

Finally we conclude that a change in the measured FSF optical thickness is mainly due to a change in mechanical layer thickness. This is caused by a variation in the smectic tilt angle $\theta$. A small discontinuity in the tilt angle exists at every phase transition between the tilted smectic phases. For the first time, we resolve a change in the smectic layer thickness of the order of $0.01 \%$ which corresponds to jumps in the tilt angle of $0.05^{\circ}$ to $0.5^{\circ}$ at the transitions between the $\mathrm{SmC}_{A}^{*}, \mathrm{SmC}_{5}$ layer (also known as $\left.\mathrm{SmC}_{A(q T=1 / 5)}^{*}[6]\right), \mathrm{SmC}_{\gamma}^{*}$ (also known as $\left.\operatorname{SmC}_{A(q T=1 / 3)}^{*}\right)$, and $\mathrm{AF}\left(\mathrm{SmC}_{A(q T=1 / 2)}^{*}\right)$ phases. A number of materials possessing these phases including MHPOBC, 12OF1M7, and its homolog have been investigated and all of them possess similar behavior. The technique has proven to be useful for detecting the intermediate phases existing over extremely narrow temperature regions such as the $\mathrm{SmC}_{5}^{*}$ layer phase in $12 \mathrm{OF} 1 \mathrm{M} 7$. The other advantage of it is that it does not require the application of an external electric field which can disturb the liquid crystalline structure.

The tilt angle is found to depend not only on the temperature, but also on the molecular arrangement (synclinic or anticlinic), leaving room for further theoretical treatment.

We thank A. Fukuda, M. A. Osipov, A. V. Emelyanenko, F. Goc, and M. Linehan for valuable discussions. SFI Grant No. 02/In.1/I.031 and IRCSET are thanked for funding the work.
[1] R. B. Meyer, L. Liebert, L. Strzelecki, and P. Keller, J. Phys. (France) 36, L69 (1975).

[2] D. Photinos and E. T. Samulski, Science 270, 783 (1995).

[3] A. D. L. Chandani, Y. Ouchi, H. Takezoe, A. Fukuda, K. Terashima, K. Furukawa, and A. Kishi, Jpn. J. Appl. Phys., Part 2 28, L1261 (1989).

[4] P. Mach, R. Pindak, A.-M. Levelut, P. Barois, H. T. Nguyen, C. C. Huang, and L. Furenlid, Phys. Rev. Lett. 81, 1015 (1998).
[5] A. V. Emelyanenko and M. A. Osipov, Phys. Rev. E 68, 051703 (2003)

[6] V. P. Panov, N. M. Shtykov, A. Fukuda, J. K. Vij, Y. Suzuki, R. A. Lewis, M. Hird, and J. W. Goodby, Phys. Rev. E 69, 060701(R) (2004).

[7] A. D. L. Chandani, N. M. Shtykov, V. P. Panov, A. V. Emelyanenko, Atsuo Fukuda, and J. K. Vij, Phys. Rev. E 72, 041705 (2005). 
[8] T. Isozaki, T. Fujikawa, H. Takezoe, A. Fukuda, T. Hagiwara, Y. Suzuki, and I. Kawamura, Jpn. J. Appl. Phys., Part 2 31, L1435 (1992).

[9] A. Fukuda, Y. Takanishi, T. Isozaki, K. Ishikawa, and H. Takezoe, J. Mater. Chem. 4, 997 (1994).

[10] K. Itoh, M. Kabe, K. Miyachi, Y. Takanishi, K. Ishikawa, H. Takezoe, and A. Fukuda, J. Mater. Chem. 7, 407 (1997).

[11] C. R. Safinya, M. Kaplan, J. Als-Nielsen, R. J. Birgeneau, D. Davidov, J. D. Litster, D. L. Johnson, and M. E. Neubert, Phys. Rev. B 21, 4149 (1980).

[12] A. V. Emelyanenko, A. Fukuda, and J. K. Vij, Phys. Rev. E 74, 011705 (2006).

[13] N. M. Shtykov, J. K. Vij, R. A. Lewis, M. Hird, and J. W. Goodby, Liq. Cryst. 28, 1699 (2001).

[14] M. Skarabot, M. Cepic, B. Zeks, R. Blinc, G. Heppke, A. V. Kityk, and I. Musevic, Phys. Rev. E 58, 575 (1998).

[15] Y. Takanishi, A. Ikeda, H. Takezoe, and A. Fukuda, Phys. Rev. E 51, 400 (1995).

[16] D. W. Berreman, J. Opt. Soc. Am. 62, 502 (1972).

[17] P. Mach, R. Pindak, A.-M. Levelut, P. Barois, H. T. Nguyen,
H. Baltes, M. Hird, K. Toyne, A. Seed, J. W. Goodby, C. C. Huang, and L. Furenlid, Phys. Rev. E 60, 6793 (1999).

[18] P. M. Johnson, D. A. Olson, S. Pankratz, T. Nguyen, J. Goodby, M. Hird, and C. C. Huang, Phys. Rev. Lett. 84, 4870 (2000).

[19] D. Konovalov, H. T. Nguyen, M. Copic, and S. Sprunt, Phys. Rev. E 64, 010704 (2001).

[20] N. M. Shtykov, J. K. Vij, and H. T. Nguyen, Phys. Rev. E 63, 051708 (2001).

[21] A. Cady, J. A. Pitney, R. Pindak, L. S. Matkin, S. J. Watson, H. F. Gleeson, P. Cluzeau, P. Barois, A.-M. Levelut, W. Caliebe, J. W. Goodby, M. Hird, and C. C. Huang, Phys. Rev. E 64, 050702(R) (2001).

[22] I. Musevic and M. Skarabot, Phys. Rev. E 64, 051706 (2002).

[23] V. P. Panov, J. K. Vij, N. M. Shtykov, S. S. Seomun, D. D. Parghi, M. Hird, and J. W. Goodby, Phys. Rev. E 68, 021702 (2003).

[24] V. P. Panov, B. K. McCoy, Z. Q. Liu, J. K. Vij, J. W. Goodby, and C. C. Huang, Phys. Rev. E 74, 011701 (2006). 